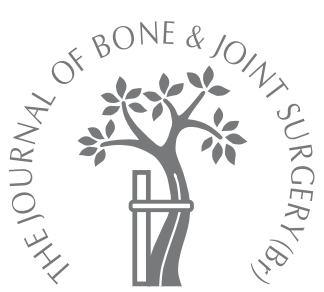

F. von Knoch, T. Böhm, M. L. Bürgi, M. von Knoch, H. Bereiter

From the Kantonsspital Chur, Switzerland

\title{
Trochleaplasty for recurrent patellar dislocation in association with trochlear dysplasia
}

\author{
A 4- TO 14-YEAR FOLLOW-UP STUDY
}

We investigated the clinical and radiological outcome of trochleaplasty for recurrent patellar dislocation in association with trochlear dysplasia in 38 consecutive patients (45 knees) with a mean follow-up of 8.3 years (4 to 14 ).

None had recurrence of dislocation after trochleaplasty. Post-operatively, patellofemoral pain, present pre-operatively in only 35 knees, became worse in 15 (33.4\%), remained unchanged in four $(8.8 \%)$ and improved in $22(49 \%)$. Four knees which had no pain preoperatively $(8.8 \%)$ continued to have no pain.

A total of 33 knees were available for radiological assessment. Post-operatively, all but two knees (93.9\%) had correction of trochlear dysplasia radiologically but degenerative changes of the patellofemoral joint developed in $30 \%(10)$ of the knees.

We conclude that recurrent patellar dislocation associated with trochlear dysplasia can be treated successfully by trochleaplasty, but the impact on patellofemoral pain and the development of patellofemoral osteoarthritis is less predictable. Overall, subjective patient satisfaction with restored patellofemoral stability after trochleaplasty appeared to outweigh its possible sequelae.

Patellofemoral instability is a common clinical problem. Risk factors predisposing to patellar dislocation include ligamentous laxity, femoral or tibial malrotation, patella alta or femoral trochlear dysplasia. ${ }^{1}$ The last has been reported to occur in up to $85 \%$ of patients with recurrent patellar dislocation. ${ }^{2}$ It is characterised by an abnormal trochlear shape which lacks congruence with the patella. ${ }^{3}$ The diagnosis is made when a true lateral radiograph of the knee demonstrates the crossing sign, the trochlear bump and a decrease in trochlear depth. ${ }^{2}$ The crossing sign refers to the floor line of the trochlear sulcus crossing the anterior border of the femoral condyles. The trochlear bump represents a prominent anterior extension of the trochlear groove in relation to the projection of the anterior cortex of the distal femur (Fig. 1).

In 1994, Bereiter and Gautier ${ }^{4}$ described a technique of trochleaplasty which involved chiselling off a trochlear osteochondral flake and deepening the subchondral bony groove with a high-speed burr. This allows delimination of the anterior bump of the trochlear floor and the possibility of remodelling of the flattened trochlear groove.

We report the clinical and radiological results of trochleaplasty after 4 to 14 years based on this technique. ${ }^{4}$

\section{Patients and Methods}

Between 1991 and 2001 the senior author (HB) performed trochleaplasty on 48 consecutive knees (40 patients). The indication for trochleaplasty was recurrent patellar dislocation in the presence of a positive Fairbank's apprehension sign ${ }^{5}$ and the confirmation of trochlear dysplasia on radiographs. Contraindications included open epiphyseal growth plates or pronounced degenerative changes of the trochlea despite patellofemoral instability in association with trochlear dysplasia. Two patients (three knees) were lost to follow-up. We therefore retrospectively reviewed the clinical and radiological outcome of 45 knees in 38 patients (16 men, 22 women) with a mean follow-up of 8.3 years (4 to 14) after trochleaplasty. The mean age of the patients at the time of surgery was 22.2 years (15 to 31 ). A total of 15 knees had undergone previous surgery, such as debridement, medial retinacular reefing, lateral retinacular release or medialisation of the tibial tuberosity.

Operative technique. The surgical procedure involves exposure of the trochlea through a lateral parapatellar approach and medial retraction of the patella with the knee in full extension. The articular cartilage is separated from the synovium, and the medial and lateral 


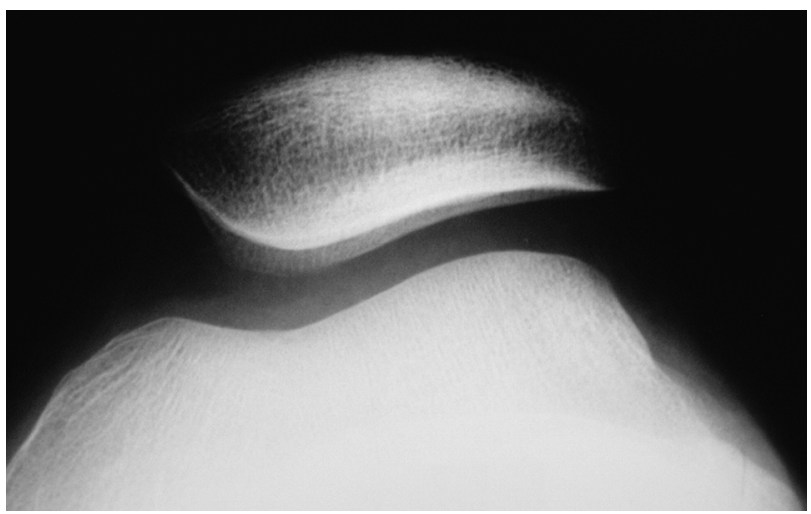

Fig. 1a

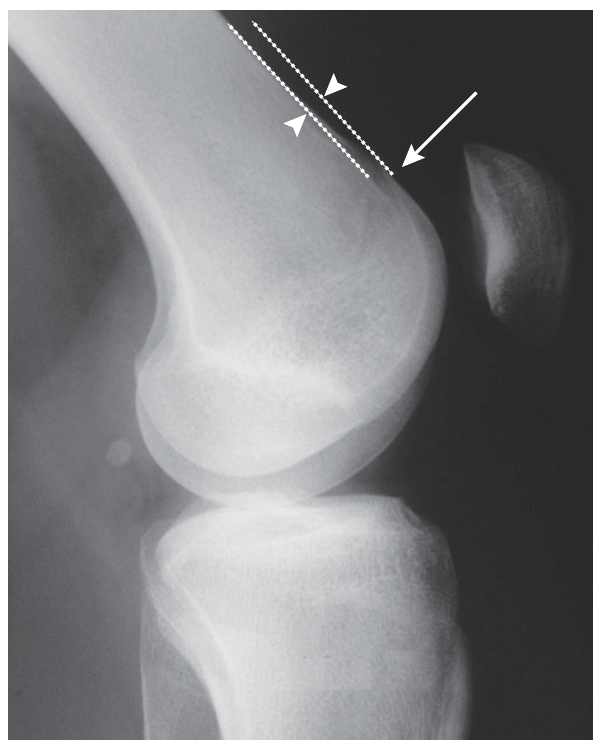

Fig. 1b

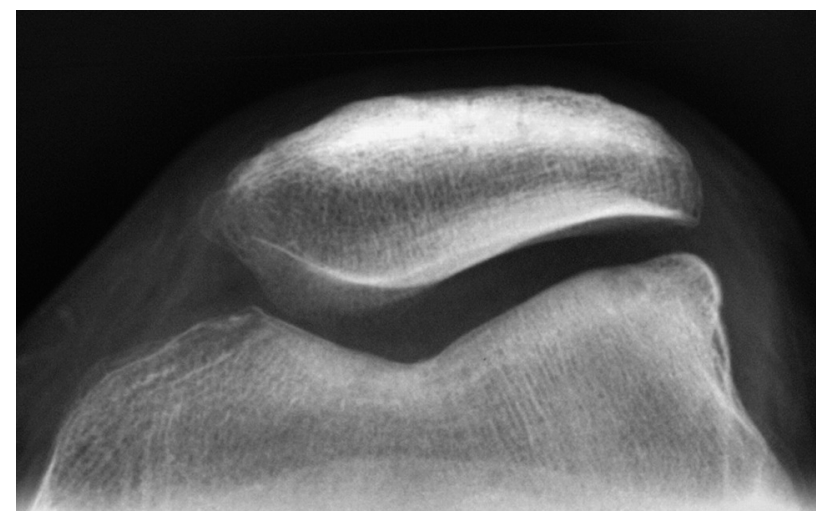

Fig. 1c

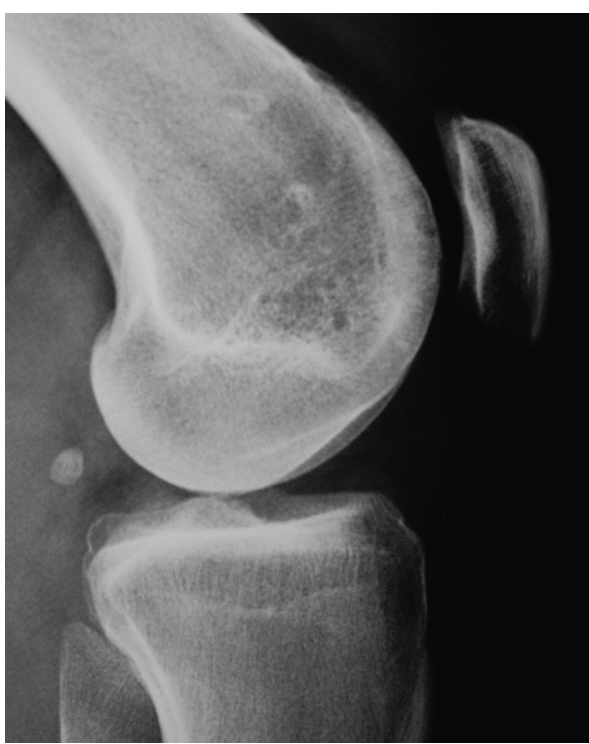

Fig. 1d

A 19-year-old woman with recurrent patellar dislocation associated with trochlear dysplasia. Pre-operative skyline (a) and lateral (b) radiographs of the knee. The positive crossing sign (arrow), the increased trochlear bump (double arrow), the decreased trochlear depth, and medialisation of the dysplastic trochlea are present. Skyline (c) and lateral (d) radiographs six years after trochleaplasty showing correction of all the radiological signs of trochlear dysplasia, and mild osteoarthritic patellofemoral changes. The patellofemoral joint was classified as grade 1 according to Iwano et al. ${ }^{7}$ There is lateralisation of the remodelled trochlear groove.

condyles are chiselled off together with the trochlea as one piece using curved osteotomes in a proximal to distal direction, to within $5 \mathrm{~mm}$ of the notch, thereby leaving $2 \mathrm{~mm}$ of subchondral bone on the distally pedunculated osteochondral flake. The subchondral groove is then deepened using a straight chisel and a high-speed burr. The trochlear osteochondral flake is subsequently seated and pressed into the newly-formed groove. This is fixed by two transosseous vicryl bands $3 \mathrm{~mm}$ wide (Ethicon Products, Norderstedt, Germany) in its deepest part in a U-shaped fashion through two $3.2 \mathrm{~mm}$ holes drilled into the cortex of the lateral femoral condyle. The synovium is re-attached to the condylar edges with a continuous absorbable suture. A medial reefing with or without reconstruction of the medial patellofemoral ligament as well as closure of the lateral retinaculum is performed as required. The joint is closed in a standard fashion by apposition of the synovium followed by skin closure. Intra-articular drainage is removed after 24 hours. Patients are mobilised in a hinged knee immobiliser with full weight-bearing. Daily knee-flexion exercises are encouraged by continuous passive movement for the duration of the hospital stay. A $20^{\circ}$ block to full extension is placed for six weeks. The rationale behind this is to centralise the patella within the remodelled intercondylar groove; thereby controlling the contact pressure of the patella on the remodelled trochlear groove to facilitate healing. Six weeks after surgery, the brace is removed and gradual implementation of a full range of movement is encouraged, with return to normal daily activities as tolerated. 
Table I. Clinical results in 45 knees with recurrent patellar dislocation associated with trochlear dysplasia at a mean of 8.3 years (4 to 14) following trochleaplasty

\begin{tabular}{lc}
\hline & Number \\
\hline Level of sporting activity post-operatively & \\
Higher & 44 \\
Lower & 1 \\
Apprehension sign & \\
$\quad$ Pre-operative & 45 \\
Post-operative & 1 \\
Patellofemoral pain & \\
Pre-operative & 35 \\
Post-operative & \\
$\quad$ Worse & 15 \\
$\quad$ Unchanged & 4 \\
$\quad$ Improved & 22 \\
$\quad$ No pain & 4 \\
Crepitus & 28 \\
Kujala score mean (range) & 94.9 (80 to 100) \\
\hline
\end{tabular}

Table II. Radiological data of 33 knees available for analysis with recurrent patella dislocation associated with trochlear dysplasia before trochleaplasty and at a mean of 8.3 years ( 4 to 14 ) after surgery

\begin{tabular}{clc}
\hline & Pre-operative & Post-operative \\
\hline Number with crossing sign (\%) & $33(100)$ & $2(6.1)$ \\
Mean trochlear depth in mm (range) & $-0.1(-7$ to 5$)$ & $5(0$ to 10$)$ \\
Mean trochlear bump in mm (range) & $3.9(0$ to 10$)$ & $0.4(-3$ to 6$)$ \\
Number (\%) with patellofemoral & & \\
osteoarthritis ${ }^{*}$ & $31(93.9)$ & $9(27.3)$ \\
Grade 0 & $2(6.1)$ & $14(42.4)$ \\
1 & 0 & $7(21.2)$ \\
2 & 0 & $2(6.1)$ \\
3 & 0 & $1(3.0)$ \\
4 & & \\
Number $(\%)$ with tibiofemoral osteo- & $28(84.8)$ \\
arthritis $^{\dagger}$ & & $4(12.2)$ \\
Grade 0 & $33(100)$ & 0 \\
1 & 0 & $1(3.0)$ \\
2 & 0 & 0 \\
\hline
\end{tabular}

* according to Iwano et $\mathrm{al}^{7}$

$\dagger$ according to Kellgren and Lawrence ${ }^{8}$

Clinical assessment. There were 45 knees available for follow-up. Clinical assessment included determination of subjective findings such as patellofemoral pain, instability, redislocation, the level of sporting activities and overall satisfaction. Objective findings such as the apprehension sign, range of movement, crepitus of the patellofemoral joint and the score of Kujala et al, ${ }^{6}$ specifically designed for patellofemoral disorders, were also recorded. Since this study is retrospective, pre-operative Kujala scores were not available.

Radiological assessment. A total of 33 knees were available for complete radiological assessment with pre-operative and post-operative radiographs which included anteroposterior, lateral and skyline patellar views at $45^{\circ}$. Radiological data for the remaining 12 knees was either incomplete or not of sufficient quality for the purpose of the study. The radiographs were analysed by an experienced radiologist (TB) for the crossing sign, the trochlear depth, the trochlear bump, ${ }^{2}$ and for the presence of osteoarthritis in the patellofemoral and tibiofemoral compartments using the classification system of Iwano et $\mathrm{al}^{7}$ and that of Kellgren and Lawrence. ${ }^{8}$

Statistical analysis. Differences between pre- and post-operative mean values were analysed by the paired Student $t$-test. All p-values were compared with an alpha-value of 0.05 to determine statistical significance.

\section{Results}

The post-operative complications in the reviewed patients were limited to patella baja in one knee, and a tendency for subluxation in another who underwent successful additional distal realignment using the Elmslie-Trillat procedure. ${ }^{9}$ There were no cases of patellar dislocation after trochleaplasty.

Details of the results are given in Table I. The positive apprehension sign which was found pre-operatively in all the patients was abolished in all but one. All the patients reported an improvement in patellofemoral stability resulting in higher levels of sporting activity except in one. It should be emphasised that an improvement in sporting activity from the patient's perspective was entirely subjective. We did not use an established clinical scoring system for this parameter.

Patellofemoral pain was present in 35 knees before surgery and was not present in 10. After trochleaplasty, pain increased in 15 knees $(33.4 \%)$, remained unchanged in four $(8.8 \%)$ and improved in $22(49 \%)$. Four knees $(8.8 \%)$ which were painfree before surgery remained so. Patellofemoral crepitus was noted in 28 knees $(62 \%)$ at final evaluation. All the knees had a full range of movement. The mean Kujala patellofemoral score was 94.9 (80 to 100) at the most recent follow-up (Table I). Overall satisfaction was assessed by asking the patients whether they would elect to undergo trochleaplasty again. All responded unequivocally and affirmatively.

The radiological assessment of the 33 knees evaluated is shown in Table II and Figure 1. Post-operatively, the crossing sign remained positive in two knees $(6.1 \%)$. The trochlear depth increased from a mean of $-0.1 \mathrm{~mm}(-7$ to +5$)$ preoperatively to $5.0 \mathrm{~mm}$ (0 to 10$)$ post-operatively (p < 0.001). The mean trochlear bump decreased from $3.9 \mathrm{~mm}$ (0 to 10$)$ pre-operatively to 0.4 (-3 to 6) post-operatively $(p<0.001)$. Of the 31 knees $(93.9 \%)$ with Iwano grade- $0^{7}$ degenerative changes of the patellofemoral joint at the time of surgery, only nine $(27.3 \%)$ showed no degenerative changes at the latest review. Fourteen knees $(42.4 \%)$ had grade-1 changes, seven $(21.2 \%)$ grade-2, two $(6.1 \%)$ grade- 3 and one $(3 \%)$ grade- 4 . There were definite osteoarthritic changes of grade- 2 or worse at the latest follow-up in ten knees $(30 \%)$ in the patellofemoral compartment. With respect to the tibiofemoral compartment, all the knees had grade- 0 changes at time of surgery and $28(84.8 \%)$ had 
grade- 0 changes, four $(12.2 \%)$ had grade- 1 and one $(3 \%)$ grade- 3 changes at follow-up.

\section{Discussion}

Trochlear dysplasia represents an important anatomical predisposition for patellar instability with recurrent patellar dislocation, ${ }^{2}$ and subsequent patellofemoral osteoarthritis. ${ }^{10}$ Trochleaplasty is a challenging surgical procedure designed to improve patellofemoral tracking by remodelling the trochlear groove. Different surgical techniques have been described in an attempt to correct trochlear dysplasia. Albee ${ }^{11}$ elevated the lateral facet of the dysplastic trochlea by an osteotomy and interposition of a tibial graft without diminishing the trochlear bump. This procedure may induce an increased pressure across the lateral facet of the patellofemoral articulation with the potential to generate patellofemoral pain and subsequently patellofemoral osteoarthritis. Trochleaplasty as variously proposed by Masse, ${ }^{12}$ Dejour et al, ${ }^{3}$ and Bereiter and Gautier, ${ }^{4}$ aimed for proximal extended reconstruction of the flattened trochlear groove which then allowed optimal patellar tracking especially during early degrees of flexion. Masse ${ }^{12}$ described a technique of cartilage impaction without fixation to the subchondral bone. Dejour et $\mathrm{al}^{3}$ reported a technique in which the trochlea was remodelled by an osteotomy of the two condyles generating a V-shape rather than its usual concave U-shape. The technique of Bereiter ${ }^{4}$ aimed to impose a more anatomically correct lateralisation and concave remodelling of the medialised dysplastic trochlea by chiselling off the trochlear cartilage using curved osteotomes. A recent report using CT analysis has confirmed these predictions. ${ }^{13}$

In the present study, trochleaplasty using this method successfully restored patellofemoral stability in a series of patients with recurrent patellar dislocation in association with trochlear dysplasia, often in spite of previous surgical intervention. No further patellar dislocation occurred in our series up to 14 years after trochleaplasty. A positive apprehension test, as found pre-operatively in all patients, was abolished in all but one. In addition to a good clinical outcome, the radiological features of trochlear dysplasia were corrected in most cases. These encouraging results are further supported by a previous report by Schottle et al, ${ }^{13}$ using Bereiter's technique, but with a shorter follow-up.

The outcome with respect to post-operative patellofemoral pain is less predictable. Patellofemoral pain, presenting before trochleaplasty in only 35 (78\%) of 45 knees, became worse in $15(33.4 \%)$ knees. The presence of established osteoarthritic changes of the patellofemoral joint in $30 \%$ (10 of 33 ) of this series suggests that these degenerative changes may at least in part be responsible for the relatively high prevalence of post-operative patellofemoral pain in the long term.

The cause of degeneration of the patellofemoral joint in nearly one-third of our patients after trochleaplasty is likely to be multifactorial. The technique itself may represent a predisposing factor for osteoarthritis as has been proposed for other patellar stabilising procedures. ${ }^{14-16}$ It may compromise the viability of the articular cartilage after surgery since the integrity of the bone-cartilage interface is subject to an iatrogenic injury pattern similar to that found in articular fractures. Furthermore, the anatomy of the patellofemoral joint after trochleaplasty is likely to be disturbed and the potential adaptation of the articular cartilage to the altered contact stresses and shear forces in the patellofemoral joint has not been thoroughly investigated. Conversely, it is known that recurrent patellar dislocations without surgical intervention is associated with damage to articular cartilage and a higher incidence of patellofemoral osteoarthritis. ${ }^{10}$ In support of these findings, we found distinct signs of patellar chondromalacia of grade-I or grade-II severity, based on Outerbridge's classification, ${ }^{17}$ in most of the knees at the time of surgery and it is likely that these were produced by previous recurrent dislocation. When Bereiter's technique was initially used, we were unaware of the relevance of this issue. Thus, precise pre-operative data on patellar chondral damage were not recorded.

We wish to stress the fact that trochleaplasty should be avoided in children with open physis since disturbance of growth is a major risk. Furthermore, in older patients a cautionary approach is warranted when there are advanced degenerative changes of the femoral trochlea because osteoarthritic femoral condyles lack sufficient cartilaginous plasticity. This may lead to an increased risk of a fracture of the osteochondral flake which may then require additional fixation. When performing trochleaplasties initially using the Bereiter technique our radio-diagnostic assessment before surgery relied primarily upon conventional radiographs. However, we now use CT for further specification and classification of trochlear dysplasia according to Dejour et al. ${ }^{2}$ MRI is used additionally to assess selectively the chondral damage of the patellofemoral articular surfaces before trochleaplasty. Furthermore, we have also modified our postoperative regime and now mobilise selected patients on the day after surgery without bracing.

Our study suggests that trochleaplasty according to Bereiter is a reasonable surgical procedure for the treatment of recurrent patellar dislocation in association with trochlear dysplasia, but may not prevent subsequent development of osteoarthritis of the patellofemoral joint. Overall, subjective patient satisfaction with restored patellofemoral stability after trochleaplasty appears to outweigh its possible sequelae.

We wish to thank Dr P. N. Perinchief for his editorial assistance.

No benefits in any form have been received or will be received from a commercial party related directly or indirectly to the subject of this article.

\section{References}

1. Arendt E. Anatomy and malalignment of the patellofemoral joint: its relation to patellofemoral arthrosis. Clin Orthop 2005;436:71-5.

2. Dejour H, Walch G, Nove-Josserand L, Guier C. Factors of patellar instability: an anatomic radiographic study. Knee Surg Sports Traumatol Arthrosc 1994;2:19-26.

3. Dejour H, Walch G, Neyrat P, Adeleine P. Dysplasia of the femoral trochlea. Rev Chir Orthop Reparatrice Appar Mot 1990;76:45-54 (in French). 
4. Bereiter H, Gautier E. Die trochleaplastik als chirurgische Therapie der rezidivierenden Patellaluxation bei Trochleadysplasie des Femurs. Arthroskopie 1994:7: 281-6.

5. Fairbank HAT. Internal derangement of the knee in children and adolescents. Proc $R$ Soc Med 1937;30:427.

6. Kujala UM, Jaakkola LH, Koskinen SK, et al. Scoring of patellofemoral disorders Arthroscopy 1993:9:159-63.

7. Iwano T, Kurosawa H, Tokuyama H, Hoshikawa Y. Roentgenographic and clinical findings of patellofemoral osteoarthrosis: with special reference to its relationship to femorotibial osteoarthrosis and etiologic factors. Clin Orthop 1990:252:190-7.

8. Kellgren JH, Lawrence JS. Radiological assessment of osteo-arthrosis. Ann Rheum Dis 1957;16:494-502.

9. Cox JS. An evaluation of the Elmslie-Trillat procedure for management of patellar dislocations and subluxations: a preliminary report. Am J Sports Med 1976;4:72-7.

10. Maenpaa H, Lehto MU. Patellofemoral osteoarthritis after patellar dislocation. Clin Orthop 1997:339:156-62.
11. Albee FH. The bone graft wedge in the treatment of habitual dislocation of the patella. Med Rec 1915;88:257-9.

12. Masse Y. Trochleoplasty: restoration of the intercondylar groove in subluxations and dislocations of the patella. Rev Chir Orthop Reparatrice Appar Mot 1978;64:3-17 (in French).

13. Schottle PB, Fucentese SF, Pfirrmann C, Bereiter H, Romero J. Trochleaplasty for patellar instability due to trochlear dysplasia: a minimum 2-year clinical and radiological follow-up of 19 knees. Acta Orthop 2005;76:693-8.

14. Arnbjornsson A, Egund N, Ryding 0, Stockerup R, Ryd L. The natural history of recurrent dislocation of the patella: long-term results of conservative and operative treatment. J Bone Joint Surg [Br] 1992;72-B:140-2.

15. Crosby EB, Insall J. Recurrent dislocation of the patella: relation of treatment to osteoarthritis. J Bone Joint Surg [Am] 1976;58-A:9-13.

16. Hampson WG, Hill P. Late results of transfer of the tibial tubercle for recurrent dislocation of the patella. J Bone Joint Surg [Br] 1975;57-B:209-13.

17. Outerbridge R. The etiology of chondromalacia patellae. J Bone Joint Surg $[\mathrm{Br}]$ 1961;43-B:752-7. 